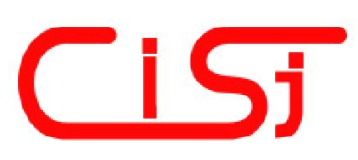

\title{
DETERMINING THE OPERATING DISTANCE OF AIR ULTRASOUND RANGE FINDERS: CALCULATIONS AND EXPERIMENTS
}

\author{
Omar S. Sonbul ${ }^{1)}$, Alexander N. Kalashnikov ${ }^{2)}$ \\ ${ }^{1)}$ Department of Computer Engineering, Umm Al-Qura University, \\ Makkah, P.O. Box: 715, Saudi Arabia, \\ e-mail: ossonbul@uqu.edu.sa \\ ${ }^{2)}$ Department of Electrical and Electronic Engineering, the University of Nottingham, \\ Nottingham, NG7 2RD, UK, \\ e-mail: Alexander.Kalashnikov@nottingham.ac.uk
}

\begin{abstract}
Estimating the operating distance of air ultrasound range finders by using the suitably modified radar equation and experimental verification of the developed computational procedure is discussed. It is shown that, despite notable differences between operating conditions of radars and air ultrasonic range finders, the radar equation is applicable to the considered case, and calculations of the relevant terms for this case are presented. The experimental assessment was carried out by evaluating the probability of detection at various distances from the custom built device. The calculated and experimental results seem to agree well despite using a number of values with high degree of uncertainty. The described procedure can be used at the design stage of air ultrasound range finders in order to reduce the number of prototypes before finalizing the design to a single prototype. Copyright (C) Research Institute for Intelligent Computer Systems, 2014. All rights reserved.
\end{abstract}

Keywords: air ultrasound, range finding, radar equation, operating distance.

\section{INTRODUCTION}

Air ultrasound range finders are used for distance measurements in construction and surveying (ultrasonic tape measures [1]), for proximity sensing [2], and for collision avoidance in parking aids [3] and autonomous vehicles [4]. Some other applications, which are being developed at the moment, include ultrasonic sensing of surface profiles [5], distributed intrusion detection systems [6] and walking aids for people with visual impairments [7].

These devices are frequently built around inexpensive ultrasonic modules, such as SRF-04 [8], which commonly operate using a pitch-catch mode, and consist of transmitting and receiving ultrasonic transducers and their supporting electronic circuitry. (Some of the modules operate in the pulse-echo mode using a single transducer only.) The electronic circuitry produces an excitation pulses when externally triggered, and returns a level change at its output when a returning signal with amplitude over the particular threshold is detected. The external controller then measures the ultrasound propagation time and calculates the distance to the object using either the known or assumed ultrasound velocity.
Meeting the design specifications of an ultrasonic range finder can be very tricky because it is not uncommon for the manufacturers and vendors of the same ultrasonic modules, to claim notably different operating distances for their products. Indeed, the same module might confidently detect the presence of a solid wall located perpendicularly to the transducer's axis, while at the same time failing to detect a typical parking pole, even at a much smaller distance. A procedure for determining the operating range for newly designed devices does not seem to be readily available publicly, which makes it difficult to meet the desired specifications without a few rounds of trials and error corrections.

On the other hand, the operating range of radars can be found using the well understood radar equation [9]. In this paper we present a procedure for determining the operating distance of air ultrasonic range finders that is based on the radar equation. We discuss qualitative and quantitative differences between the two a.m. devices, and show how to calculate the required parameters based on datasheets of typical ultrasonic transducers. Calculated results are compared to the experimental ones that are obtained by detecting a man at different distances to a custom built air ultrasound range 
finder which features various transducers and excitation voltages.

\section{COMPARING RADAR OPERATION TO THAT OF THE AIR ULTRASOUND RANGE FINDER}

Active radars operate by sending electromagnetic waves towards objects that reflect some of the wave energy back. These waves are generated by the transmitter and then radiated into the space using an antenna. Next, some of the transmitted energy is scattered in various directions by the target. These echoes, reflected back towards the radar, are collected by the receiving antenna and passed on to the receiver for further processing, which commonly includes detection of the object's presence and evaluation of the distance to it, if detection has occurred.

The operating range of radar can be found from the radar equation, which was derived under the following major assumptions [9]:

1) If high-frequency energy is emitted by an isotropic transmitter, then it spreads uniformly in all the directions; therefore, areas with the same power density will form spheres where the transmitted energy is distributed evenly across the area $A=4 \pi R_{a}^{2}$, where $\mathrm{R}_{\mathrm{a}}$ is the distance to the antenna;

2) If the radiated power is redistributed to provide better radiation in the particular direction of interest, then this results in an increase of the power density in this direction proportional to the antenna's directional gain $\mathrm{G}$;

3) Both the transmitter and receiver use the same antenna with the same G;
4) The echo power depends upon the transmission power density at the target position and how much of it is reflected back in the direction of the receiver. It is equal to the product of the power density at the target and its effective radar cross-section (scattering coefficient of the target) $\sigma$;

5) The echo power spreads out in the same way as the transmitted power (i.e., its power density is inversely proportional to $\mathrm{R}_{\mathrm{a}}{ }^{2}$ ). When propagation of both the transmitted and reflected waves is considered, then the returned power density thus becomes inversely proportional to $\mathrm{R}_{\mathrm{a}}^{4}$.

The radar equation, derived from these assumptions, is given below [9]

$$
R_{a}=\sqrt[4]{\frac{P_{s} * G^{2} * \lambda^{2} * \sigma}{P_{E^{*}}(4 \pi)^{3} * \text { Lges }}},
$$

where $P_{S}$ is the transmitted power, $\lambda$ is the radar wavelength at the operating frequency, $\mathrm{P}_{\mathrm{E}}$ is the received power that is sufficient in order to detect the target, and $\mathrm{L}_{\text {ges }}$ is the loss factor that covers propagation losses. $\mathrm{P}_{\mathrm{E}}$ equals to the product of the echo power density at the receiver's antenna and its effective area.

Qualitatively, the operations of radar and air ultrasonic range finder are similar to each other. Pitch-catch operating mode, commonly employed for air ultrasonic range finders, is preferred over the pulse-echo mode because of the lower dimension of the dead zone.

Quantitatively, the differences between the two came from the common operating conditions of these devices (Table 1).

Table 1. Typical relations between the operating parameters

\begin{tabular}{|c|c|c|c|c|}
\hline & $\begin{array}{l}\text { Distance to the } \\
\text { target }\end{array}$ & $\begin{array}{c}\text { Linear size of the } \\
\text { target }\end{array}$ & $\begin{array}{l}\text { Linear size of } \\
\text { the antenna }\end{array}$ & $\begin{array}{c}\text { Operating } \\
\text { wavelength } \lambda\end{array}$ \\
\hline $\begin{array}{c}\text { Radar } \\
\left(c=3 * 10^{8} \mathrm{~m} / \mathrm{s}\right) \\
\end{array}$ & $\begin{array}{c}\mathrm{R}_{\mathrm{a}} \gg> \\
\text { tens-hundreds km }\end{array}$ & $\begin{array}{c}\mathrm{L}_{\mathrm{o}} \approx \\
\text { tens-hundreds } \mathrm{m}\end{array}$ & $\begin{array}{c}\mathrm{L}_{\mathrm{a}}>> \\
\text { tens-hundreds } \mathrm{m}\end{array}$ & $\begin{array}{c}30 \mathrm{~mm} \text { typical } \\
\text { (US airport radar @ } 10 \mathrm{GHz})\end{array}$ \\
\hline $\begin{array}{l}\text { Air ultrasonic } \\
\text { range finder } \\
(c=340 \mathrm{~m} / \mathrm{s})\end{array}$ & $\begin{array}{l}\mathrm{R}_{\mathrm{a}} \approx \\
\text { few m }\end{array}$ & $\begin{array}{l}\mathrm{L}_{\mathrm{o}}>> \\
\text { few m }\end{array}$ & $\begin{array}{c}\mathrm{L}_{\mathrm{a}} \approx \\
\text { tens } \mathrm{mm}\end{array}$ & $\begin{array}{l}8 \mathrm{~mm} \text { typical } \\
(\text { @ } 40 \mathrm{kHz})\end{array}$ \\
\hline
\end{tabular}

The most important difference comes from the fact that radar operates at much higher distances compared to the sizes of the objects and antennas, whilst air ultrasonic range finders are equipped with a tiny antenna compared to the operating distance and size of the target. Despite this, both devices operate in the far field of the antenna, which suggests that their respective operating distance equations should be similar.

Let us consider the validity of the assumptions, which lead to the radar equation, but now for air ultrasonic range finders. Air ultrasonic transducers are usually characterized not by their directional gain but by their radiating angle. It is assumed that all of the transmitted ultrasound energy is spread inside this angle evenly. Therefore assumptions 1 and 2 above need to be adjusted as appropriate. Assumption 3 remains valid since the two ultrasonic transducers, typical to air ultrasonic range finders, are commonly used in exactly the same way as the single radar antenna. Assumption 4 remains valid since the effective radar cross-section allows for reducing an arbitrarily complex surface profile to a 
single number. However, when this concept is applied to air ultrasonic range finders, the substantial size of the object, which is commensurable to the distance to the target, may complicate theoretical considerations of backscatter compared to the radar case. Assumption 5 describes the excitation and echo propagation as spherical waves, which should at least hold as the first order approximation for air ultrasonic range finders.

Overall, the radar equation derived to quantify the propagation of electromagnetic waves over substantial distances seems to be suitable for the case of air ultrasonic range finders provided that its terms are calculated correctly.

\section{DETERMINING TERMS OF THE RADAR EQUATION FOR AIR ULTRASOUND RANGE FINDERS}

\subsection{TRANSMITTED POWER PS}

The power of acoustic (including ultrasonic) waves can be calculated from their pressure, $p$ :

$$
P_{S}=\frac{p^{2} \times A}{Z},
$$

where $A$ is the area where the pressure is applied and $\mathrm{Z}$ is the acoustic impedance of the propagation medium $(Z=\rho c$, where $\rho$ is the air density and $c$ is the sound velocity in air, $1.19 \mathrm{~kg} / \mathrm{m}^{3}$ and $346.6 \mathrm{~m} / \mathrm{s}$ at $25^{\circ} \mathrm{C}$ respectively [10, sect. 2.1.1 and 2.4.1]).

Ultrasonic transducers are commonly characterized by their standard pressure level (SPL, $\mathrm{dB}$ ) produced under the excitation voltage of $10 \mathrm{~V}_{\mathrm{RMS}}$ relative to the reference pressure of $20 \mu \mathrm{Pa}$. From this definition, the transmitted acoustic pressure, $p_{t}$, equals to

$$
p_{t}=\frac{V_{R M S}}{10 V} \times 20 \mu P a \times 10^{S P L / 20},
$$

where $\mathrm{V}_{\mathrm{RMS}}$ is the excitation voltage. The SPL is stated at some distance from the ultrasonic transducer (typically $30 \mathrm{~cm}$ ). The area, A, at which the pressure is applied, can be approximated from the total beam angle, $\alpha$, of the transducer (Fig. 1):

$$
A=\pi \times(0.3 m \times \tan (\alpha / 2))^{2},
$$

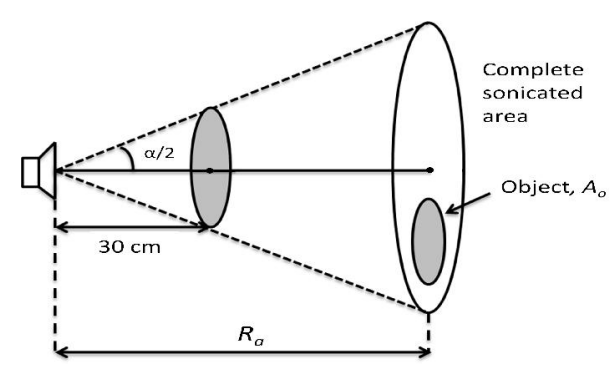

Fig. 1 - Spatial distribution of the transmitted energy.

\subsection{DIRECTIONAL GAIN G}

The gain of a round antenna or transducer can be calculated from its area $A_{t}\left(A_{t}=\pi d 2 / 4, d\right.$ is the diameter) and operating wavelength $\lambda[9]$ :

$$
G=\frac{4 \times \pi \times A_{t} \times K_{a}}{\lambda^{2}},
$$

where $K_{a}$ is the efficiency of the transmitting/receiving transducers (the typical value for ultrasonic transducers is $30 \%$ [11]). The radar equation assumes that transmission and reception are undertaken using the same antenna in the pulse-echo mode. Despite having two separate transducers in the pitch-catch configuration for air ultrasonic modules, they commonly have the same diameters. If this is not the case, then the radar equation's term $G^{2}$ should be replaced with $G_{T} \times G_{R}$, where $G_{T}$ and $\mathrm{G}_{\mathrm{R}}$ are the directional gains of the transmitting and receiving transducers respectively.

\subsection{EFFECTIVE RADAR CROSS SECTION $\sigma$}

Generally this factor is very difficult to calculate for real objects; consequently, its value for radar is usually approximated by some value measured from similar objects or by combining simulated and measured data [12]. In the case of air ultrasonic range finders, the calculations can be even more complicated, e.g., because of the varying shapes of human bodies. Here, we suggest using the cross-sectional area of the object that belongs to the sonicated area, $A_{o}$ (Fig. 1), as the first order approximation.

\subsection{RECEIVED POWER SUFFICIENT FOR THE DETECTION OF THE OBJECT $P_{E}$}

This parameter is receiver-specific as it depends upon the noise level at its input, type of transmitted signal (e.g., sine wave burst or chirp) and processing algorithm (e.g., use of matched filtering before threshold detection). The transducer should generate the minimum voltage, $V_{D}$, required to make object detection happen. The receive sensitivity of the ultrasonic transducers, $\mathrm{S}$, is commonly stated in $\mathrm{dBs}$ relative to a $1 \mathrm{~V} / \mu$ bar level. Therefore the acoustic pressure at the receiver, $\mathrm{p}_{\mathrm{r}}$, required for object detection should be at least

$$
p_{r}=\frac{V_{D}}{10^{S / 20}}\left[\frac{\mu b a r}{V}\right]=0.1 \frac{V_{D}}{10^{S / 20}}\left[\frac{P a}{V}\right]
$$

Finally, the sought after $\mathrm{P}_{\mathrm{E}}$ can be calculated from equation (2) using the area of the transducer, $\mathrm{A}_{\mathrm{t}}$, and air acoustic impedance, $\mathrm{Z}$. 


\subsection{LOSS FACTOR, $L_{\text {ges }}$}

This term includes all of the losses experienced by the wave during its propagation. The attenuation of ultrasound in the air, $\alpha$, at $20^{\circ} \mathrm{C}$ and $101.325 \mathrm{kPa}$ strongly depends upon humidity, ranging from $0.46 \mathrm{~dB} / \mathrm{m}$ to $1.3 \mathrm{~dB} / \mathrm{m}$ [10, section 2.4.1].

Inclusion of the attenuation term into the radar equation would affect the linearity of the latter because the attenuation depends upon the as-of-yet unknown radar range. For this reason, we first calculated the operating range assuming $L_{\text {ges }}=1$, and then calculate the corrected value, applying the losses during propagation in both directions, and using both the minimal and maximal $\alpha$ :

$$
R_{a}^{\prime}=\frac{R_{a}}{\sqrt[4]{\left(10^{\alpha * R} a\right)^{2}}}=\frac{R_{a}}{\sqrt{10^{\alpha * R a}}}
$$

This correction overestimates the losses for $\mathrm{R}_{\mathrm{a}}^{\prime}$, thus giving the bottom boundary for the operating distance.

\section{CALCULATING THE EXPECTED OPERATING RANGE FOR A PARTICULAR ULTRASONIC RANGE FINDER}

The calculations (and later experimental assessment) were carried out for a pulse-echo air ultrasonic range finder developed in our laboratory [13]. We select $0.6 \mathrm{mV}$ for the output of the transducer during reception as the object detection threshold voltage.

Two excitation voltage levels $\left(10 \mathrm{~V}_{\mathrm{RMS}}\right.$ and $20 \mathrm{~V}_{\mathrm{RMS}}$ ) and two different transducers (Table 2) were used, which yielded four different options for the range finder operation in the pulse-echo mode.

We detected a man standing at various distances from the transducer in an axial direction; man's cross-sectional area, $\mathrm{A}_{0}$, was approximated by a value of $0.75 \mathrm{~m}^{2}$ (from a rectangle made of the $0.5 \mathrm{~m}$ effective width and $1.5 \mathrm{~m}$ effective height).

The calculated values for the 400PT160 transducer excited by $20 \mathrm{~V}_{\mathrm{RMS}}$ were as follows: $\mathrm{P}_{\mathrm{S}}=0.150 \mathrm{~W}, \quad \mathrm{G}=10.4, \quad \sigma=0.75 \mathrm{~m}^{2}, \quad \mathrm{P}_{\mathrm{E}}=5.74 \mathrm{nW}$, $\mathrm{L}_{\text {ges }}=1$, yielding $\mathrm{R}_{\mathrm{a}}=2.99 \mathrm{~m}$.

Table 2. The specifications of the ultrasonic transducers taken from their datasheets $[14,15]$

\begin{tabular}{|l|c|c|}
\hline & $400 \mathrm{PT} 120$ & $400 \mathrm{PT} 160$ \\
\hline Diameter (mm) & 12.7 & 16.2 \\
\hline $\begin{array}{l}\text { Transmitted sound pressure } \\
\text { level (SPL) (per 10 } \mathrm{V}_{\text {rms }} \text { applied } \\
\text { at } 30 \mathrm{~cm} \text { axial distance from the } \\
\text { centre of the transducer) }\end{array}$ & $115 \mathrm{~dB}$ & $117 \mathrm{~dB}$ \\
\hline Receive sensitivity & $-68 \mathrm{~dB}$ & $-65 \mathrm{~dB}$ \\
\hline Total beam angle (@, -6 dB) & $85^{\circ}$ & $55^{\circ}$ \\
\hline
\end{tabular}

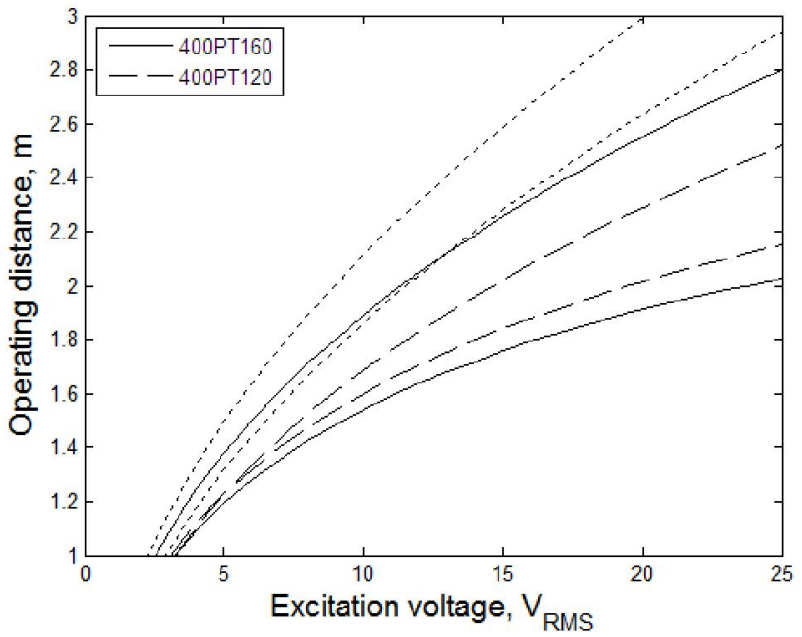

Fig. 2 - Operating distances versus the excitation voltage for two different transducers under the minimal and maximal ultrasound attenuation in air (dotted lines show distances without accounting for attenuation).

Table 3. The detection range of a person with a crosssectional area of $0.75 \mathrm{~m}^{2}$

\begin{tabular}{|c|c|c|}
\hline \multirow{2}{*}{$\begin{array}{c}\text { Operating } \\
\text { conditions }\end{array}$} & $\begin{array}{c}\text { Calculated operating range } \\
\text { without } \\
\text { attenuation } R_{a}\end{array}$ & $\begin{array}{c}\text { with attenuation } \\
R_{a}^{\prime}\end{array}$ \\
\hline $\begin{array}{c}\text { 400PT120@ } \\
10 \mathrm{~V}\end{array}$ & $1.86 \mathrm{~m}$ & $1.69 \mathrm{~m} / 1.54 \mathrm{~m}$ \\
\hline $\begin{array}{c}\text { 400PT120@ } \\
\text { 20V V }\end{array}$ & $2.63 \mathrm{~m}$ & $2.29 \mathrm{~m} / 1.91 \mathrm{~m}$ \\
\hline $\begin{array}{c}\text { 400PT160@ } \\
10 \mathrm{~V}\end{array}$ & $2.11 \mathrm{~m}$ & $1.89 \mathrm{~m} / 1.60 \mathrm{~m}$ \\
\hline $\begin{array}{c}\text { 400PT160@ } \\
\text { 20V V }\end{array}$ & $2.99 \mathrm{~m}$ & $2.55 \mathrm{~m} / 2.02 \mathrm{~m}$ \\
\hline
\end{tabular}

The calculated operating distances versus the excitation voltage for all of the considered operating conditions are plotted in Fig. 2. It displays the effect of overestimation of losses under the applied procedure - under maximal losses the operating distance of the 400PT160 transducer became even smaller than that of the 400PT 120 one.

Numerical values for calculated distances under all of the operating conditions that were tested experimentally are presented in Table 3 to enable later comparison with the experimental data.

\section{EXPERIMENTAL ASSESSMENT OF THE OPERATING RANGE OF THE DEVICE}

During the experiments a particular transducer was placed at around $1 \mathrm{~m}$ above ground, and was directed towards a man who faced the transducer [13]. 1000 ultrasonic pulses were generated with a $100 \mathrm{~ms}$ delay between each other, and the number of instances that led to detection of the echo was recorded. The probability of object 
detection was estimated as the ratio of the number of detections to the number of trials (1000). After every measurement, the man walked a further $0.5 \mathrm{~m}$ away from the transducer and this process was repeated several times. The estimated detection probabilities are plotted in Fig. 3 using spline interpolation between the experimentally derived points. Crosses show the operating distances, calculated for the lowest and highest ultrasound attenuation (right column of Table 3). The curves have a range of distances where the object was detected with confidence (if probability is close to 1 , then correct detection), and a range of distances where the object was not detected (if probability is below 0.1 , then object missed). Between these two ranges the probability of detection decreases smoothly with increasing distance as one would expect. All of the theoretically calculated operating ranges of the device (Table 3, right column) were found to belong to the transient region of the curves presented in Fig. 3 . We believe that this region is where they ought to belong to if the theoretical calculations were correct.

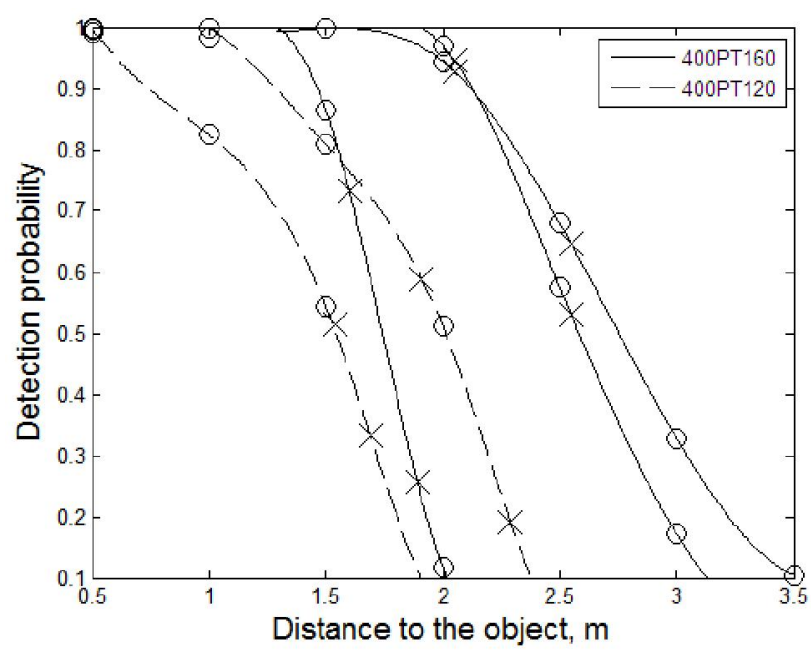

Fig. 3 - Detection probabilities for various operating conditions (curves for the $20 \mathrm{~V}_{\mathrm{RMS}}$ excitation voltage are to the right to these for the $10 \mathrm{~V}_{\mathrm{RMS}}$ excitation; circles depict the experimentally estimated points; crosses are placed on the curves at the distances taken from the right column of Table 3; experiment with $16 \mathrm{~mm}$ transducer excited by $20 \mathrm{~V}_{\mathrm{RMS}}$ was conducted twice hence two curves).

\section{DISCUSSION}

\subsection{WAS IT POSSIBLE TO INCREASE THE ACCURACY OF THE ABOVE CALCULATIONS?}

The following factors could be taken into the account:

- the size of the transducer's piezoelement is smaller (typically by about 10\%) than the diameter of the transducer; this correction would affect the transducer's area, and hence, gain;

- more accurate estimation would involve solving the nonlinear equation with the loss factor depending upon the operating distance;

- a better match between the calculated and experimental data would be expected if the relative humidity was measured at the time of the experiment in order to use the actual ultrasound attenuation in air.

However, because of significant uncertainty regarding some of the parameters used for calculations (e.g., ultrasound attenuation in air, the transducer's efficiency, etc.), we believe that there was no need for more accurate calculations.

\subsection{WHAT FACTORS, IMPORTANT}

\section{TO AIR ULTRASOUND RANGE FINDERS,}

\section{WERE LIKELY TO BE OVERLOOKED}

IN THE CALCULATIONS?

These likely were:

- scattering of ultrasound waves over the object with dimensions commensurate with the operating distance; this factor alone could have probably varied by an order of magnitude, depending on the profile of the object, compared to the flat rectangle used in the calculations;

- the limited bandwidth of the ultrasonic transducer(s) was not considered; it would spread out both the radiated and received waveforms in the time domain, most likely leading to some reduction in the operating distance;

- the radar equation does not account for the statistical nature of signal detection; the calculated distance will only be effective for particular values of detection and false alarm probabilities, which are valid for the $\mathrm{P}_{\mathrm{E}}$ value used in the calculations.

\subsection{HOW MAY THE DESCRIBED CALCULATION PROCEDURE BE BEST USED DURING THE DESIGN?}

Most of the terms that are involved in the calculations can only be evaluated with substantial uncertainty. For this reason this procedure should only be used as a very rough estimate at the first round of the design. The designed prototype is then to be evaluated experimentally, and the operating conditions (e.g., transducer types, excitation voltage) are to be adjusted accordingly in order to meet specifications. It seems that using the described calculation procedure will enable the completion of the design after a single prototyping stage. 


\section{SUMMARY AND CONCLUSIONS}

The compatibility of the well-established radar equation with air ultrasound range finders was analysed first. Then, the relevant parameters were obtained from the typical datasheets for ultrasonic transducers and other available data. The operating range of a particular air ultrasound range finder was calculated for a set of various operating conditions, and was compared to the experimental results. Despite the many uncertain values that were involved in the calculations, the experimental and calculated results agreed well.

Therefore, the radar equation is applicable to the case of air ultrasound range finders provided that its terms are calculated appropriately.

The developed numerical procedure seems to be capable of reducing the required number of prototypes before finalizing the design to a single prototype.

The operating distance, as commonly stated by the manufacturers of air ultrasound range finding modules and devices, can be very misleading. It should instead be estimated experimentally, and include the references (a) to the target (e.g., a solid wall in the direction perpendicular to the module's axis), (b) to the relative humidity at the time of measurements and (c) to the probability of target detection (at least 0.90 or 0.95 ) in order to eliminate any unreasonable expectations for performance.

\section{REFERENCES}

[1] Intellimeasure ${ }^{\mathrm{TM}}$ ultrasonic distance estimator, Product Specification, available online on tinyurl.com/mf4j6x8, accessed June 2014.

[2] Ultrasonic proximity sensors, Allen-Bradley catalogue, available online on tinyurl.com/m4hek18, accessed June 2014.

[3] Parking sensors, Wikipedia, available online on tinyurl.com/ycreypt, accessed June 2014.

[4] J. Borenstein and Y. Koren, Obstacle avoidance with ultrasonic sensors, IEEE Journal of Robotics and Automation, (2) 4 (1991), pp. 213-218.

[5] O. Sonbul, P. Popejoy and A. N. Kalashnikov, Ultrasonic sensor array for remote sensing of profiles of bulk materials, Proceedings of the IEEE International Instrumentation and Measurement Technology Conference (I2MTC), Graz, Austria, (May 13-16, 2012), pp. 1794-1797.

[6] O. Sonbul and A. N. Kalashnikov, Low cost ultrasonic wireless distributed security system for intrusion detection, Proceedings of the IEEE International Conference on Intelligent Data Acquisition and Advanced Computing
Systems, Berlin, Germany, (September 12-14, 2013), pp. 235-238.

[7] Ultracane - an award winning mobility aid, Product Description, available online on tinyurl.com/mbvqzp5, accessed June 2014.

[8] SRF04 - ultrasonic ranger, Technical specification, available online on tinyurl.com/bs3dlah, accessed June 2014.

[9] M. Skolnik, Introduction to radar systems, 3rd ed. McGraw-Hill, Boston, Mass, 2001.

[10] G. W. C. Kaye and T. H. Laby, Tables of Physical and Chemical Constants, $16^{\text {th }}$ ed., Longman, 1995.

[11] T. Kojima, H. Haya, K. Minegishi, and R. T. Nguyen, Ultrasonic velocity measurement for analysis of brick structure, Proceedings of the IEEE Ultrasonics Symposium, 2008, pp. 398-401.

[12] I. M. Martin, M. A. Alves, G. G. Peixoto and M. C. Rezende, Radar cross section measurements and simulations of a model airplane in the X-band, Piers Online, (4) 5 (2009), pp. 377-380, available online on tinyurl.com/mcoeo9y, accessed June 2014.

[13] O. Sonbul and A. N. Kalashnikov, Electronic architecture for air coupled ultrasonic pulseecho range finding with application to security and surface profile imaging, Universal Journal of Electrical \& Electronic Engineering, (3) 2 (2014), pp. 105-111.

[14] Air ultrasonic ceramic transducers 400PT120, datasheet, available online on tinyurl.com/kwgm4wc, accessed June 2014.

[15] Air ultrasonic ceramic transducers 400PT160, datasheet, available online on tinyurl.com/n9hwqjc, accessed July 2014.

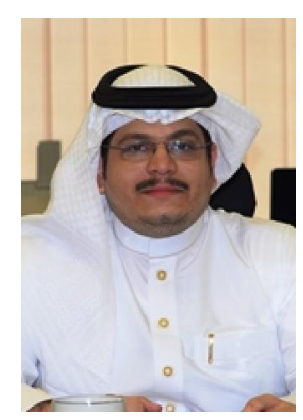

Dr Omar S. Sonbul was born in 1980 in Makkah, Saudi Arabia. $\mathrm{He}$ received his $B$.Sc. degree in 2003 from Umm Al-Qura University in Makkah, Saudi Arabia. Then, he completed his M.Sc.degree at the University of Nottingham, UK, in 2008. In 2012, he obtained his Ph.D. degree from the University of Nottingham by a thesis entitled "Intrusion Detection and Profile Imaging using Networked Electronic Modules for Air Coupled Ultrasonic Transducers". Dr.Sonbul is currently an Assistant Professor in the Department of Computer Engineering at Umm AlQura University. His research focuses on the development of low-cost ultrasonic electronic modules for air coupled applications. 


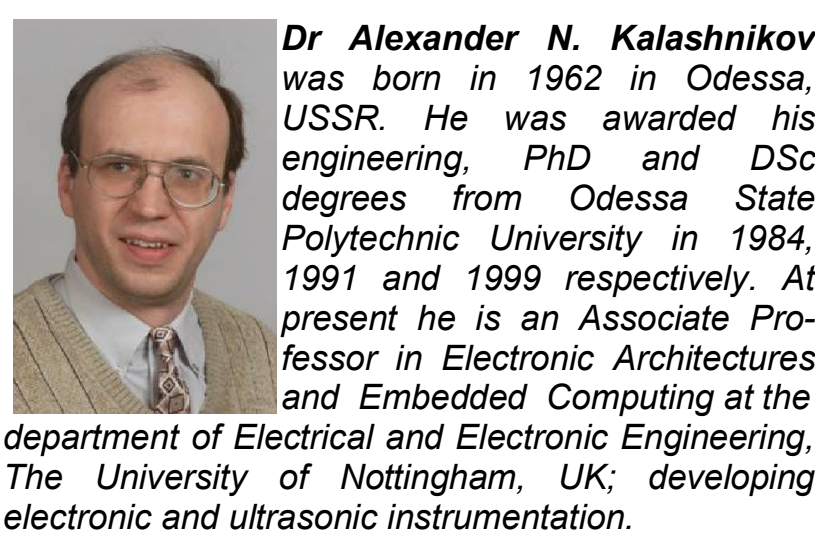

